\title{
Correction to: Computational Examination of Synaptic Plasticity and Metaplasticity in Hippocampal Dentate Granule Neurons
}

Azam Shirrafiardekani, Jörg Frauendiener, Ahmed A. Moustafa, and Lubica Benuskova

\section{Correction to:}

Chapter 20 in: V. Cutsuridis et al. (eds.), Hippocampal Microcircuits, Springer Series in Computational Neuroscience, https://doi. org/10.1007/978-3-319-99103-0_20

In the original version of this chapter, two of the chapter authors were inadvertently missed to be added in the authors list. The authors' names Jörg Frauendiener, and Lubica Benuskova have now been added in chapter 20. 